\title{
Destination and source memory in Huntington's disease
}

\author{
Mohamad El Haj ${ }^{1 *}$, Marie Caillaud ${ }^{2}$, Christophe Verny ${ }^{3,4}$, \\ Luciano Fasotti ${ }^{5}$ and Philippe Allain ${ }^{2,4}$
}

'Research Unit on Cognitive and Affective Sciences, Department of Psychology, University of North of France, Lille, France

${ }^{2}$ UNAM University, Psychology Laboratory (EA 4638), University of Nantes and Angers, France

${ }^{3}$ UMR CNRS 62 I4, Inserm UI083, University of Angers, France

${ }^{4}$ Neurology Department, $\mathrm{CHU}$ of Angers, France

${ }^{5}$ Donders Institute for Brain, Cognition and Behaviour, Radboud University Nijmegen, the Netherlands

\begin{abstract}
Destination memory refers to the recall of the destination of previously relayed information, and source memory refers to the recollection of the origin of received information. We compared both memory systems in Huntington's disease (HD) participants. For this, HD participants and healthy adults had to put 12 items in a black or a white box (destination task), and to extract another 12 items from a blue or a red box (source task). Afterwards, they had to decide in which box each item had previously been deposited (destination memory), and from which box each item had previously been extracted (source memory). HD participants showed poorer source as well as destination recall performance than healthy adults in the proposed tasks. Correlation analysis showed that destination recall was significantly correlated with episodic recall in HD participants. Destination memory impairment in HD participants seems to be considerably influenced by their episodic memory performance.
\end{abstract}

Until recently, little was known about destination memory or the ability to remember the receiver of transmitted information (Gopie \& MacLeod, 2009). This memory can be compared by analogy for the ability to remember the source from which specific information was acquired, termed as source memory (Schacter, Kaszniak, Kihlstrom, \& Valdiserri, 1991).

According to Gopie and MacLeod (2009), destination memory and source memory are both part of the episodic memory system initially described by Tulving (2002). Gopie and MacLeod (2009) state that destination memory and source memory are episodic in nature, as these memory components are important for the recollection of the context (e.g., the time and place) in which information has been presented, with reference to oneself as a participant in the zepisode. However, for these authors (Gopie \& MacLeod, 2009), destination memory and source memory are fundamentally different in terms of the

*Correspondence should be addressed to Mohamad El Haj, Département de Psychologie, Université de Lille 3, Domaine du Pont de Bois, 59653 Villeneuve d'Ascq, France (email: mohamad.elhaj@univ-lille3.fr). 
direction of information transfer; output in the case of destination memory and input in the case of source memory.

In comparison with source memory, destination memory has been found to be more prone to forgetting. This issue was investigated by Gopie and MacLeod (2009) who asked younger adults, in a destination memory task, to tell facts associated with pictures of famous peoples and to decide, in a later recognition test, whether they had previously told facts belong to the corresponding pictures or not. In another source memory task, the participants were given facts associated with pictures of famous peoples and, on a later recognition test, they had to decide whether they had previously received the facts from the corresponding pictures or not. These tasks resulted in greater difficulties in destination than in source memory. Gopie, Craik, and Hasher (2010) also found this pattern of performance in healthy older adults.

In patients with brain damage, the ability to recall the context of learnt information has been shown to be dissociable from the recollection of the learnt information itself. Schacter, Harbluk, and McLachlan (1984) were among the first to observe this phenomenon in individuals with frontal lobe lesions using a source memory paradigm. In their work, individuals with frontal lobe damage were asked to read statements that included fictional information about celebrities (e.g., 'Bob Hope's father was a fireman'). After a few minutes, the participants were able to correctly answer questions concerning the newly learnt facts, but they had great difficulties remembering where the information had been initially acquired. On the basis of further observations, Schacter et al. (1984) found a source memory deficit in his patients which was also associated with impairment in a test of mental flexibility. Following this suggestion and the historical association between the prefrontal cortex and executive processes (Fuster, 1997; Shallice \& Burgess, 1991), source failures have been generally attributed to executive dysfunctions and/or to frontal lobe impairment (Craik, Morris, Morris, \& Loewen, 1990; Duarte, Ranganath, \& Knight, 2005; Schacter et al., 1984; Simons et al., 2002).

Compared to source memory, destination memory has received little attention in individuals suffering from brain damage. In fact, only three studies can be found in the literature (El Haj, Moroni, Luyat, Omigie, \& Allain, 2014; El Haj, Postal, \& Allain, 2013; El Haj, Postal, Le Gall, \& Allain, 2013). In these studies, younger adults, older adults, and individuals with mild Alzheimer disease (AD) were asked either to tell facts or to imagine telling facts to pictures of famous people. Three major findings emerged from these studies. First, destination memory performance in AD participants was significantly lower than in older adults, which in turn was lower than in younger adults. Second, significant correlations were found between the performance on destination memory tasks and several executive measures of inhibition, flexibility, and binding. Third, among the executive measures, inhibition emerged as a main factor predicting variance in destination memory performance. Noteworthy was also that individuals with AD tend to show greater difficulties on source memory tasks than healthy elderly controls (Barba, Nedjam, \& Dubois, 1999; El Haj, Fasotti, \& Allain, 2012a; Goldman, Winograd, Goldstein, O'jile, \& Green, 1994; Multhaup \& Balota, 1997).

In the present study, we intend to further explore the consequences of neurodegenerative lesions on destination and source memory performance. Our aim was twofold: (1) to examine the possibility of an acquired destination memory impairment in individuals with Huntington's disease (HD) with presumed deficits in episodic memory and executive functioning, originating from cortical and sub-cortical atrophy mainly concerning the striatum and its prefrontal connections (Henley et al., 2008; Kassubek, Gaus, \& Landwehrmeyer, 2004; Rosas et al., 2002), and (2) to look at the 
possible relationship between destination/source memory, executive functioning and episodic performance and in these participants.

Clinically, HD is characterized by motor symptoms (chorea, rigidity, and abnormal posturing) occurring in mid-adulthood (Harper, 1996). Although these symptoms are the most obvious manifestation of HD, there is evidence for subtle cognitive and behavioural abnormalities preceding the motor symptoms (Snowden, Craufurd, Thompson, \& Neary, 2002). Cognitive impairment in these individuals encompasses several domains, mainly including executive functions (Craufurd \& Snowden, 2002), episodic memory (El Haj, Caillaud, Fasotti, Verny, \& Allain, 2013; Solomon et al., 2007), and other cognitive functions such as attention, language or recognition of facial emotions (see for example Stout et al., 2011).

In the case of executive functions, many studies have shown that HD participants performed poorly on tasks such as the Wisconsin Card Sorting (Jason et al., 1997), the Stroop task (Craufurd \& Snowden, 2002), verbal fluency (Jason et al., 1997), Tower of Hanoi-style problems (Lawrence et al., 1996), and the Trail Making Task (Bachoud-Lévi et al., 2001), all tasks which are sensitive to prefrontal cortical lesions. These impairments suggest an overall global impairment of cognitive executive functioning in HD.

As to memory, a large body of empirical studies has revealed that episodic memory failures in HD largely arise due to strategic or organizational failures at the time of information acquisition and retrieval, not because of a primary disorder of retention (Bachoud-Lévi et al., 2001; Jason et al., 1997; Lawrence et al., 1996; Stout et al., 2011; Verny et al., 2007). However, a meta-analysis (Montoya et al., 2006) of studies examining episodic memory performance in HD indicates that both recognition and recall abilities are impaired in HD. In a recent paper, El Haj, Caillaud et al. (2013) have suggested that HD participants tend to suffer from a perturbation in associative or integrative mechanisms responsible for the combination of different contextual features into complex episodic representations. To our knowledge, only two studies have examined contextual memory in HD participants using source memory tasks. Brandt and colleagues (Brand, Bylsma, Aylward, Rothlind, \& Gow, 1995) presented HD participants and healthy adults with true and made-up facts about celebrities. In a later recognition phase, participants were again presented with the previous made-up facts, but mixed with distractors that were not proposed in the study phase. Participants had to answer questions related to each fact (i.e., item memory), and had to decide whether each fact had been previously encountered in the test phase or not (i.e., source memory). Although displaying normal item memory performance, HD participants showed significant deterioration in source performance. The latter outcome was confirmed by Fine et al. (2008) who presented HD and $\mathrm{AD}$ participants with two lists of 16 words, each taken from the California Verbal Learning Test-Second Edition. In a later recognition phase, participants had to remember in which list the words had been presented. Compared with AD participants, HD participants demonstrated poorer source memory.

A limitation of the studies assessing context memory in HD (Brand et al., 1995; Fine et al., 2008) is that they only assessed one contextual aspect, namely source memory. In addition, executive functions were poorly investigated in these studies. In the study of Brand et al. (1995), only a limited number of executive processes (i.e., inhibition and flexibility) were tested, and the study by Fine $e t$ al. (2008) did not include these processes. Finally, as recognized by Fine et al. (2008), the California Verbal Learning Test does not assess a specific component memory process, such as source memory, as precisely as experimental paradigms designed exclusively for that purpose do. Hence, the aforementioned studies present several limitations and it may be assumed 
that they do not exhaustively assess context memory and its relations with episodic memory and executive functioning in HD. To address this shortcoming, the present paper assessed source and destination components of context memory as well as executive functioning and episodic functioning in HD participants early in the course of the disease with several specific experimental tasks. These performances were compared with those of healthy adults, matched for age, gender, and years of education. Our main research questions were: (1) Are there destination and source memory impairments in individuals with HD? (2) If destination and source deficits can be shown in individuals with HD, are they more severe in destination, in source or in both memory systems?; and (3) Are impairments in destination and source memory performance associated with executive and/or episodic memory disorders in HD participants?

\section{Method}

\section{Participants}

Fifteen participants with clinically diagnosed and genetically confirmed (mild to moderate) HD and 18 healthy adults took part in this study as volunteers. The HD participants $(7$ women and 8 men, mean age $=45.67, S D=9.04$; mean number of schooling years $=11.39, S D=1.62$ ) received annual medical and neuropsychological monitoring in the Department of Neurology of the University Hospital of Angers. They were screened for symptoms of HD using the sub-scales derived from the Unified Huntington's Disease Rating Scale (UHDRS; Huntington Study Group, 1996). These subscales include a motor and a functional scale, assessing the symptomatology of the disease. The motor scale assesses motor symptoms like chorea, speech, oculomotor, and gait impairments, as rated on a $0-4$ point scale $(4=$ most impaired $)$. This $(0-4)$ scale represents the diagnostic confidence rating, representing the evaluator's confidence that the presence of motor abnormalities is a manifestation of HD. The mean rating of HD participants was $23.62(S D=17.87)$. The total score on the motor scale ranges from 0 to 124 points and higher scores are suggestive of increased disability. The mean rating of HD participants on this sub-scale was $13.75(S D=3.76)$.

The mean illness duration of HD participants was 5.7 years $(S D=3.6)$ and their mean Cytosine-Adenine-Guanine repeats was $44.93(S D=2.82)$. Nine participants were under psychiatric medication (i.e., Venlafaxine) to address mood changes and/or anxiety, which are commonly observed in HD. Five of these participants were also under neuroleptic medication (e.g., Tiapride). Seven participants were treated with medication to reduce involuntary movements (e.g., Xenazine). Two participants were taking sleep medication (e.g., Temesta).

Most healthy adults $(9$ women and 9 men, mean age $=48.78, S D=13.34$; mean number of schooling years $=13.22, S D=3.02$ ) were spouses of the participants. They showed no evidence of neuropsychological impairment or neurological or psychiatric disease. Healthy adults and HD participants did not differ significantly in terms of age, $t$ $(31)=1.48, p>.05$, gender, $\chi^{2}(1, N=33)=0.04, p>.05$, or educational level, $t$ (31) $=0.77, p>.05$.

\section{Background neuropsychological assessment}

Background neuropsychological assessment included a measure of general cognitive functioning (Mattis Dementia Rating Scale, MDRS; Mattis, 1976), an episodic memory test (the Hopkins Verbal Learning Test-Revised, HVLT-R; Shapiro, Benedict, Schretlen, \& 
Brandt, 1999), and executive assessment of inhibition, shifting, updating, and spontaneous flexibility (for more details about this neuropsychological battery, see El Haj \& Allain, 2012; El Haj, Fasotti, \& Allain, 2012a,b; El Haj, Postal, \& Allain, 2013; El Haj, Postal, \& Le Gall et al., 2013; El Haj, Caillaud et al., 2013; El Haj, Allain, \& Kessels, 2014; El Haj, Moroni et al., 2014).

The neurological, psychiatric, and neuropsychological assessments were performed by experienced clinicians. Consistent with the Declaration of Helsinki, all participants gave written informed consent after receiving a detailed explanation of the study.

\section{Destination memory and source memory assessment}

Twenty-four everyday life items (e.g., comb, watch, brush, etc.) were selected according to their occurrence in French language (Content, Mousty, \& Radeau, 1990). These items were normal-sized and without distinctive features. They were randomly assigned to each of two sets: destination memory set and source memory set. Four $20 \times 20 \mathrm{~cm}$ boxes were also used: (1) a black and a white box for the destination task, and (2) a red and a blue box for the source task. Figure 1 summarizes the destination and source tasks.

The destination task included three phases: a study phase, an interpolate, and a recognition phase. In the study phase, participants were informed about the real goal of the study, that is, their memory for the items and their association with the boxes would be tested in a later phase of the experiment. They were subsequently presented with items of the destination memory set and were asked to deposit half of the items in the black box and the other half in the white box. In order to ensure encoding, they had to name each item before associating it to a correspondent destination. Note that the participants were
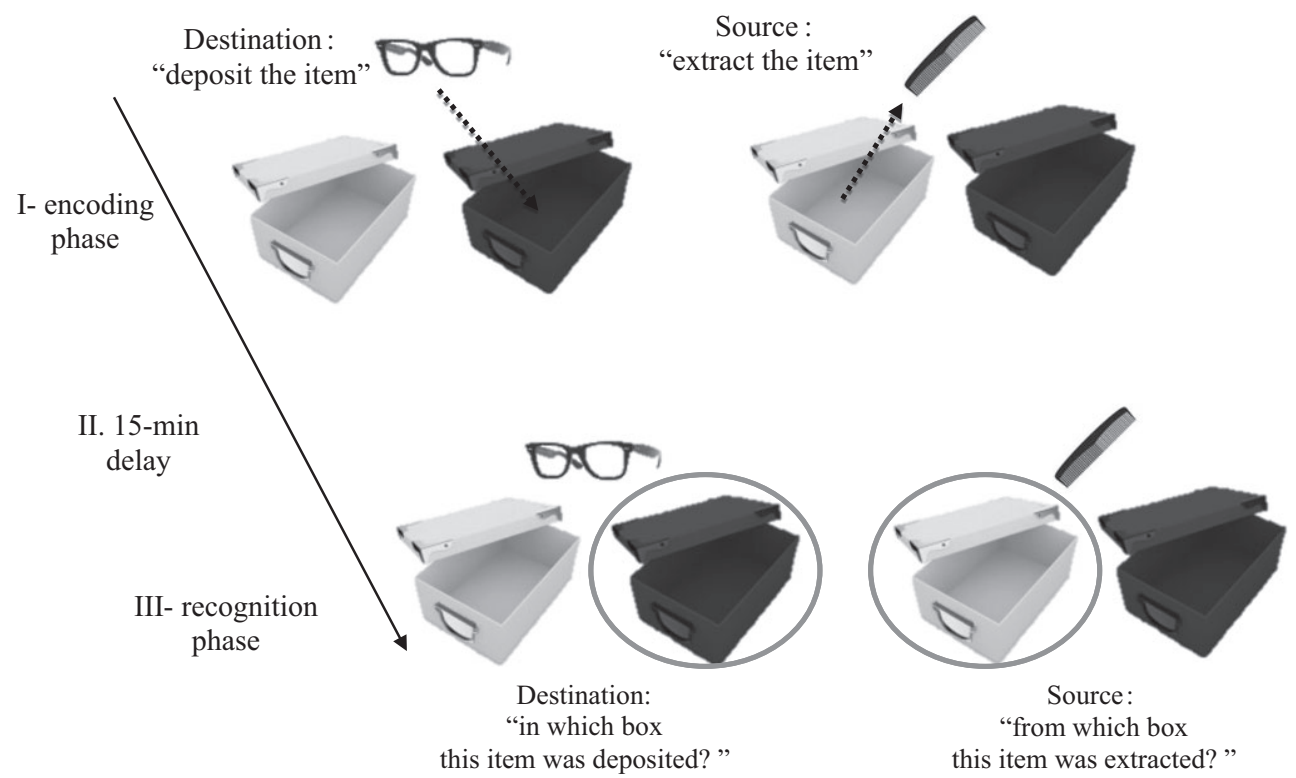

Figure I. Illustration of the destination and source tasks. Participants had to deposit I 2 items in a black or a white box (destination memory). They also had to extract another 12 items from another two boxes. After 15-min delay, they had to decide in/from which box each item had previously been deposited/ extracted. 
free to associate items to their destination; they only had to deposit six items in each box. Once all items were put away in the boxes, the participants were engaged in an interpolated activity lasting $15 \mathrm{~min}$, and consisting of neuropsychological testing. This phase was immediately followed by the recognition phase. Herein, the experimenter presented items of the destination memory set, one item at a time, and asked the participants to decide whether the item had previously been deposited in the black or in the white box.

The source memory task was identical to the destination one. However, the participants were presented with a red and a blue box, each box containing half of the items from the source memory set. The participants had to extract, name, and put aside the 12 items, one item at a time. After the interpolated phase, they were presented with the items of source memory set and had to decide, for each one, whether it had previously been extracted from the blue or from the red box.

The source and destination tasks were counterbalanced across participants, destination memory set, and source memory set were counterbalanced across tasks, and the same can be said for the boxes. The number of correct responses was the dependent variable.

\section{Results}

\section{Statistical analysis}

Table 1 shows the performances of the HD patients and controls on the neuropsychological and experimental tests.

Prior to performing these analyses, all variables were plotted and checked for normal distribution with the Shapiro-Wilk test. For between-group comparisons, when statistical distribution was normal, Student's $t$-tests were performed, otherwise, non-parametric Wilcoxon signed rank-sum test were used due to the small sample sizes. As no significant differences were found between groups with respect to age and education level, these variables were not entered as covariates in correlational analyses. For subsidiary analyses, where indicated, raw scores were converted to $Z$-scores. Additionally correlative analyses

Table I. Performances of HD participants and healthy adults

\begin{tabular}{lccc}
\hline & $\mathrm{HD}(n=15)$ & Controls $(n=18)$ & Cohen's d \\
\hline General Cognitive functioning: MDRS & $136.47(5.23)^{* * *}$ & $143.89(.32)$ & 2.01 \\
Episodic memory: HVLT-R & $19.80(4.36)^{* * *}$ & $27.33(3.27)$ & 1.95 \\
$\quad$ & & & \\
immediate free recall & $77.03(26.14)^{* *}$ & $50.21(20.11)$ & 1.17 \\
Inhibition: Stroop & $17.86(14.05)^{*}$ & $9.54(4.55)$ & .77 \\
Shifting: Plus-Minus & $8.33(5.08)^{*}$ & $5.00(1.94)$ & .78 \\
Spdating: 2-back & $17.47(6.45)^{*}$ & $23.61(6.56)$ & .94 \\
Destination memory flexibility: verbal fluency & $8.93(1.90)^{* *}$ & $10.61(1.35)$ & 1.02 \\
Source memory & $9.27(1.91)^{* *}$ & $11.00(1.08)$ & 1.11 \\
\hline
\end{tabular}

Note. Standard deviations are given in parenthesis. MDRS, Mattis Dementia Rating Scale; HVLT-R, Hopkins Verbal Learning Test-Revised; HD, Huntington disease. Scores on the Stroop and Plus-Minus tasks refers to reaction time; scores on the 2-back task refer to the number of erroneous responses; scores on the verbal fluency task refers to the number of correctly generated words; scores on the destination and source tasks refer to the number of correct responses. 
were conducted using Spearman's rank correlation. For all tests, the level of significance was set at $p<.05$.

\section{Neuropsychological assessment: poor episodic and executive performance in HD participants}

Student's $t$-tests showed poorer performance in HD participants than healthy adults on the MDRS, $t(31)=6.02, p<.001$. The same can be said about episodic performance, as represented by the total immediate free recall score on the HVLT-R task, $t(31)=5.66$, $p<.001$. Student's $t$-tests also showed significantly poorer performance in HD participants than healthy adults on the inhibition, $t(31)=3.12, p<.01$, the shifting, $t$ $(31)=2.42, p<.05$, the updating, $t(31)=2.62, p<.05$, and the spontaneous flexibility tasks, $t(31)=2.64, p<.05$.

\section{Poor destination and source recognition in HD participants}

The Shapiro-Wilk test, used due to the small sample sizes, showed a non-normal distribution for destination $(\phi<.05)$ and source $(\phi<.001)$ scores. Therefore, these data were analysed as non-parametric data.

Mann-Whitney $U$-tests showed group differences in destination, $U=65.00, p<.01$, and source recognition, $U=55.00, p<.01$. In other words, HD participants showed poorer destination and source recognition than healthy adults. However, the Wilcoxon signed rank-sum test did not reveal significant differences between destination and source recognition, neither for healthy adults, $z=-1.62, p>.05$, nor for HD participants, $z=-.44, p>.05$.

In order to threshold whether HD individual participants were impaired on destination and source tasks, performances were converted to $Z$-scores using the control data mean and standard deviations. Then performances were considered to be affected if $Z$-scores were below -1.96 . Performances of $12 \mathrm{HD}$ participants were compromised in the destination memory task, and performances of 10 participants were compromised in the source memory task. Significant differences were detected between the number of HD participants with compromised destination memory ( $n=12$ participants) and those with normal performances $\left(n=3\right.$ participants), $\chi^{2}(1, N=15)=5.40, p<.05$, but no significant differences were detected between participants with compromised source memory ( $n=13$ participants) and those with normal performances ( $n=5$ participants), $\chi^{2}(1, N=15)=1.67, p>.05$. No significant differences were detected between HD participants with compromised destination memory ( $n=3$ participants) and those with compromised source memory $(n=5$ participants $), \chi^{2}(1, N=30)=.68, p>.05$.

\section{Significant correlations between destination and episodic memory in HD participants} The three episodic memory scores (total immediate free recall, delayed free recall, and delayed recognition) were transformed into $Z$-scores, and the three $Z$-scores were averaged into a total $Z$-score. The same procedure was performed for the executive scores (inhibition, shifting, updating, and spontaneous flexibility), the destination performance scores, and the source performance scores. Table 2 shows the correlations between episodic $Z$-score, executive $Z$-score, and destination and source performance scores in each sample. 'Destination memory and episodic performance were significantly correlated in HD participants, while all remaining correlations were found to be no significant. 
Table 2. Correlation matrix for episodic memory, executive function, destination, and source memory performances in HD participants and healthy adults

$\begin{array}{llll}\text { I. Episodic memory } & \text { 2. Executive functions } & \text { 3. Destination memory } & 4 \text {. Source memory }\end{array}$

\begin{tabular}{rrrrr}
\hline $\begin{array}{c}\text { Controls } \\
\text { I }\end{array}$ & - & & \\
2 & -.20 & - & \\
3 & .40 & -.37 & .31 & - \\
4 & .32 & .16 & & \\
$\mathrm{HD}$ & & & & - \\
$\mathrm{I}$ & - & - & - &. \\
2 & -.11 & -.42 & .11 & - \\
3 & $.64^{*}$ & .19 & \\
4 & .21 & &
\end{tabular}

Note. Performances refer to Z-scores. HD, Huntington's disease. ${ }^{*}$ The correlation coefficient is significant at $p<.05$.

\section{Discussion}

The main aim of the present paper was to investigate destination and source memory in HD participants. Another objective was assessing the relationship between destination memory in HD participants and their executive and/or episodic memory performances. The results reveal several findings. First, HD participants showed poorer destination and source recognition than healthy adults. However, no significant differences were found between destination and source memory in both groups. In other words, HD participants showed similar performances in the source and destination tasks, and the same thing can be said for healthy adults. Second, destination recognition in HD participants was significantly correlated with episodic performance.

In line with previous studies evaluating destination memory in $\mathrm{AD}$ participants (El Haj et al., 2012a; El Haj, Postal, \& Allain, 2013; El Haj, Postal, \& Le Gall et al., 2013; El Haj, Caillaud et al., 2013; El Haj, Allain et al., 2014; El Haj, Moroni et al., 2014), our HD participants showed a significant deterioration of destination recall; a result that can be attributed to HD-related episodic memory impairment e. As shown by our analyses, destination recognition in HD participants was significantly correlated with their episodic memory performance. Noteworthy is that episodic impairment can be considered as an early marker of cognitive deterioration in the disease (Pirogovsky et al., 2009). One consideration, based on previous research, is that there are robust impairments in free recall, cued recall, and recognition performances in symptomatic HD participants, suggesting deterioration in both encoding and retrieval processes (for a review, see, Montoya et al., 2006). Episodic deterioration can also be observed in pre-symptomatic gene-carriers of HD. Duff et al. (2010), for instance, found mild episodic impairment in individuals who were estimated to be over 14 years from a motor diagnosis of HD. Taken together, these studies suggest significant deterioration of episodic memory in HD, a consideration that may account for the impairment of destination memory in our HD participants. The relationship between destination and episodic memory can be explained by looking at the contextual aspects of destination memory. Destination memory implies the recollection of the episodic context in which information was relayed. In other words, this memory subsystem implies the evaluation of the contextual 
characteristics that give past experiences of their episodic features in our subjective experience. In this view, destination memory can be embedded into the episodic memory system (for the same view, see, Gopie \& MacLeod, 2009), hence it is not surprising that destination memory deterioration may be related to episodic memory impairment e. Another common feature between destination memory and episodic memory is that both memories require association between information and its encoding context. This associative process has been widely considered as a crucial element of episodic recall, for instance, studies suggest that accurate episodic recall depends on an associative process during encoding (i.e., binding) connecting the context features to the central event (Mitchell \& Johnson, 2009). By this view, destination memory impairment, as observed in our HD participants, can be attributed to difficulties in binding information to its destination (for a same view, see, El Haj, Allain et al., 2014).

In a similar way, HD participants showed impairments in source memory; a result that confirms several studies showing source memory impairment in HD (Brand et al., 1995; Fine et al., 2008). According to Fine et al. (2008), problems with source memory in HD can be attributed to damage in the fronto-striatal circuitry connecting the striatum to the frontal lobes, as observed in the disease. More generally, the frontal lobes are known to play a key role in source memory. Individuals with frontal lesions show significant deterioration in source recall and recent fMRI work emphasizes the involvement of dorsolateral prefrontal cortex (dorsal BA 10, BA 46, and BA 9) in the control processes of source memory, such as the organization and the coupling of multiple features to their correspondent source (for a review, see, Mitchell \& Johnson, 2009). Moreover, a recent fMRI study evidenced reduced blood-oxygen-level dependent activity in the dorsolateral prefrontal cortex in preclinical HD mutation carriers (Wolf et al., 2011), providing a plausible neuroanatomical substrate for source memory deterioration in the disease.

Although showing deterioration in destination and source-recognition memory, our HD participants did not show significant differences between these recognition processes, a pattern also observed in the healthy adults. This result seems counterintuitive when considering the studies showing greater deterioration of destination than source recall in younger as well as in older adults (Gopie \& MacLeod, 2009; Gopie et al., 2010). These apparently contradictory findings can be reconciled by shedding light on a crucial difference between our destination and source tasks. In the destination task, the participants were free to associate the items and their destination, whereas such an association was predetermined in the source task. Being more involved in generating the destination than the source, our participants were likely to benefit more from cognitive and sensorimotor cues during source recollection than when remembering destination. This may explain their better performance in the destination memory task. The benefits of active engagement in destination memory, as we tend to suggest, fits with the well-known generation effect. This effect explains why better recall can be observed for self-generated information compared to passively received information (Mulligan, Lozito, \& Rosner, 2006; Slamecka \& Graf, 1978). The memory advantage produced by self-generation has been the extensively investigated, showing generation benefits on a wide variety of stimulus material (e.g., words, sentences, numbers, and pictures) in a wide variety of memory tasks (e.g., free recall, cued recall, and recognition; for review, see, Wolf et al., 2011). This fits well with the attribution of our participants' better destination task performance to their active engagement. Self-generation is likely to alleviate the difference between destination and source memory, as reported by Gopie and MacLeod (2009) and Gopie et al. (2010). Active engagement, characterizing our destination memory task, can be considered as a limitation of our study as it points to a methodological difference 
between our destination and source tasks. When considered from this viewpoint, our results can be attributed to the differences between active and passive engagement rather than to differences between destination and source performance per se. Noteworthy however is that associating information with a given destination (e.g., 'shall I tell John or Philippe about my promotion?') implies a more unconstrained choice than receiving information from a given source (e.g., 'my director told me about my promotion'). A difference neglected by most studies on destination memory, in which the correspondence between information and destination was predetermined and participants had no choice but to passively accept this attribution (El Haj, Postal, \& Allain, 2013; El Haj, Postal, \& Le Gall et al., 2013; El Haj, Allain et al., 2014; El Haj, Moroni et al., 2014; Gopie \& MacLeod, 2009; Gopie et al., 2010). The core point here is that active engagement, as characterizing our destination memory task, may account for the absence of significant differences between the destination and source performances in our participants. As we suggest, the absence of a control active source memory task or a passive destination memory task, as is the case in most destination memory studies (El Haj, Postal, \& Allain, 2013; El Haj, Postal, \& Le Gall et al., 2013; El Haj, Allain et al., 2014; El Haj, Moroni et al., 2014; Gopie \& MacLeod, 2009; Gopie et al., 2010), can be considered as a limitation of our paper. However, it is noticeable that source evaluations are typically passive (e.g., who told me about that information?).

Our results also showed a significant relationship between destination and episodic memory in HD participants. No such pattern was detected for executive functions. A substantial body of research has shown an important executive deterioration in HD participants (Bachoud-Lévi et al., 2001; Lawrence et al., 1996; Snowden et al., 2002), and this executive deterioration has been found to be related to memory impairment in HD (El Haj, Caillaud et al., 2013). In the light of the latter finding, and the small sample size of the present study, some caution is needed in ruling an association between destination memory and executive function in HD.

Analysis of individual profiles of HD participants showed average destination memory performance in three participants. Although significant differences were detected between the number of these participants and those with compromised destination memory (12/15), these outcomes suggest that not all HD participants present destination memory impairment. This outcome fits with heterogeneity of memory profiles in individuals with HD, a pattern that can be supported by some inconsistency in studies evaluating recognition memory in HD (for a critical review, see, Montoya et al., 2006). From a clinical perspective, individuals with HD tend to show some heterogeneity of memory performances, a consideration that is supported by the fact that no significant differences were detected between participants with compromised source memory and those with normal performances. However, no firm conclusion can be drawn about individual differences between HD participants on source/destination memory because of our relative small sample size, which may be considered as an important limitation of the present study. Heterogeneity of memory profiles in individuals with HD needs to be examined more in depth in future studies with large samples.

To summarize, destination memory allows the successful association between the information and its receiver, avoiding the attribution of information to an inappropriate destination and consequently developing erroneous expectations. Another consequence of destination memory distortions is redundancy, or the tendency to repeat the same information to the same receiver, as observed in individuals with neurodegenerative lesions. These consequences illustrate the impairments that destination memory impairment in HD may bring forth, impairments that merit further exploration. 


\section{Acknowledgements}

Dr. El Haj is supported by the LABEX (excellence laboratory, program investment for the future) DISTALZ (Development of Innovative Strategies for a Transdisciplinary approach to Alzheimer disease).

\section{References}

Bachoud-Lévi, A. C., Maison, P., Bartolomeo, P., Boissé, M. F., Dalla Barba, G., Ergis, A. M., ... Peschanski, M. (2001). Retest effects and cognitive decline in longitudinal follow-up of participants with early HD. Neurology, 56, 1052-1058. doi:10.1212/wnl.56.8.1052

Barba, G. D., Nedjam, Z., \& Dubois, B. (1999). Confabulation, executive functions, and source memory in Alzheimer's disease. Cognitive Neuropsychology, 16, 385-398.

Brand, J., Bylsma, F. W., Aylward, E. H., Rothlind, J., \& Gow, C. A. (1995). Impaired source memory in Huntington's disease and its relation to basal ganglia atrophy. Journal of Clinical and Experimental Neuropsychology, 17, 868-877. doi:10.1080/01688639508402436

Content, A., Mousty, P., \& Radeau, M. (1990). BRULEX: Une base de données lexicales informatisée pour le français écrit et parlé [BRULEX: A computerized lexical database for the writing and speaking of French]. L'Année Psychologique, 90, 551-566.

Craik, F. I., Morris, L. W., Morris, R. G., \& Loewen, E. R. (1990). Relations between source amnesia and frontal lobe functioning in older adults. Psychology and Aging, 5, 148-151.

Craufurd, D., \& Snowden, J. (2002). Neuropsychological and neuropsychiatric aspects of Huntington's disease. Oxford Monographs on Medical Genetics, 45, 62-94.

Duarte, A., Ranganath, C., \& Knight, R. T. (2005). Effects of unilateral prefrontal lesions on familiarity, recollection, and source memory. The Journal of Neuroscience, 25, 8333-8337. doi:10.1523/JNEUROSCI.1392-05.2005

Duff, K., Paulsen, J., Mills, J., Beglinger, L. J., Moser, D. J., Smith, M. M., . . Harrington, D. L. (2010). Mild cognitive impairment in prediagnosed Huntington disease. Neurology, 75, 500-507. doi:10.1212/WNL.0b013e3181eccfa2

El Haj, M., \& Allain, P. (2012). What do we know about the relationship between source monitoring deficits and executive dysfunction? A literature review. Neuropsychological Rehabilitation, 22, 329-488. doi:10.1080/09602011.2012.658267

El Haj, M., Allain, P., \& Kessels, R. P. (2014). The cognitive and neuroanatomical underpinnings of destination memory. Translational Neuroscience, 5, 147-151. doi:10.2478/s13380-014-0219-5

El Haj, M., Caillaud, M., Fasotti, L., Verny, C., \& Allain, P. (2013). Episodic memory decline in Huntington's disease, a binding deficit? Journal of Huntington's Disease, 2, 305-313. doi:10.3233/JHD-130056

El Haj, M., Fasotti, L., \& Allain, P. (2012a). Source monitoring in Alzheimer's disease. Brain and Cognition, 80, 185-191. doi:10.1016/j.bandc.2012.06.004

El Haj, M., Fasotti, L., \& Allain, P. (2012b). The involuntary nature of music-evoked autobiographical memories in Alzheimer's disease. Consciousness and Cognition, 21, 238-246. doi:10.1016/j.concog.2011.12.005

El Haj, M., Moroni, C., Luyat, M., Omigie, D., \& Allain, P. (2014). To what extent does destination recall induce episodic reliving? Evidence from Alzheimer's disease. Journal of Clinical and Experimental Neuropsychology, 36, 127-136. doi:10.1080/13803395.2013.869309

El Haj, M., Postal, V., \& Allain, P. (2013). Destination memory in Alzheimer's disease: When I imagine telling Ronald Reagan about Paris. Cortex, 49, 82-89. doi:10.1016/j.cortex.2011.11.014

El Haj, M., Postal, V., Le Gall, D., \& Allain, P. (2013). Destination memory in mild Alzheimer's disease. Behavioural Neurology, 26, 215-216. doi:10.3233/BEN-2012-129014

Fine, E. M., Delis, D. C., Wetter, S. R., Jacobson, M. W., Hamilton, J. M., Peavy, G., . . Salmon, D. P. (2008). Identifying the "source" of recognition memory deficits in participants with 
Huntington's disease or Alzheimer's disease: Evidence from the CVLT-II.Journal of Clinical and Experimental Neuropsychology, 30, 463-470. doi:10.1080/13803390701531912

Fuster, J. M. (1997). The prefrontal cortex: Anatomy, physiology, and neuropsychology of the frontal lobe. New York: Lippincott-Raven.

Goldman, W. P., Winograd, E., Goldstein, F. C., O'jile, J., \& Green, R. C. (1994). Source memory in mild to moderate Alzheimer's disease. Journal of Clinical and Experimental Neuropsychology, 16, 105-116. doi:10.1080/01688639408402621

Gopie, N., Craik, F. I., \& Hasher, L. (2010). Destination memory impairment in older people. Psychology and Aging, 25, 922-928. doi:10.1037/a0019703

Gopie, N., \& MacLeod, C. M. (2009). Destination memory: Stop me if I've told you this before. Psychological Science, 20, 1492-1499. doi:10.1111/j.1467-9280.2009.02472.x

Harper, P. (1996). Huntington's disease. London, UK: Saunders.

Henley, S., Wild, E. J., Hobbs, N. Z., Warren, J. D., Frost, C., Scahill, R. I., .. . Tabrizi, S. J. (2008). Defective emotion recognition in early HD is neuropsychologically and anatomically generic. Neuropsychologia, 46, 2152-2160. doi:10.1016/j.neuropsychologia.2008.02.025

Huntington Study Group (1996). Unified Huntington's Disease Rating Scale: Reliability and consistency. Huntington Study Group. Movements Disorders, 11, 136-142. doi:10.1002/mds. 870110204

Jason, G. W., Suchowersky, O., Pajurkova, E. M., Graham, L., Klimek, M. L., Garber, A. T., \& PoirierHeine, D. (1997). Cognitive manifestations of Huntington disease in relation to genetic structure and clinical onset. Archives of Neurology, 54, 1081-1088. doi:10.1001/archneur.1997. 00550210019008

Kassubek, J., Gaus, W., \& Landwehrmeyer, G. B. (2004). Evidence for more widespread cerebral pathology in early HD: An MRI-based morphometric analysis. Neurology, 62, 523-524. doi:10.1212/wnl.62.3.523-a

Lawrence, A. D., Sahakian, B. J., Hodges, J. R., Rosser, A. E., Lange, K. W., \& Robbins, T. W. (1996). Executive and mnemonic functions in early Huntington's disease. Brain, 119, 16331645.

Mattis, S. (1976). Mental status examination for organic mental syndrome in the elderly patient. Geriatric Psychiatry, 11, 77-121.

Mitchell, K. J., \& Johnson, M. K. (2009). Source monitoring 15 years later: What have we learned from fMRI about the neural mechanisms of source memory? Psychological Bulletin, 135, 638677. doi:10.1037/a0015849

Montoya, A., Pelletier, M., Menear, M., Duplessis, E., Richer, F., \& Lepage, M. (2006). Episodic memory impairment in Huntington's disease: A meta-analysis. Neuropsychologia, 44, 1984-1994. doi:10.1016/j.neuropsychologia.2006.01.015

Mulligan, N. W., Lozito, J. P., \& Rosner, Z. A. (2006). Generation and context memory. Journal of Experimental Psychology: Learning, Memory, and Cognition, 32, 836-846. doi:10.1037/0278-7393.32.4.836

Multhaup, K. S., \& Balota, D. A. (1997). Generation effects and source memory in healthy older adults and in adults with dementia of the Alzheimer type. Neuropsychology, 11, 382-391. doi:10.1037/0894-4105.11.3.382

Pirogovsky, E., Goldstein, J., Peavy, G., Jacobson, M. W., Corey-Bloom, J., \& Gilbert, P. E. (2009). Temporal order memory deficits prior to clinical diagnosis in Huntington's disease. Journal of the International Neuropsychological Society, 15, 662-670. doi:10.1017/S1355617709990427

Rosas, H. D., Liu, A. K., Hersch, S., Glessner, M., Ferrante, R. J., Salat, D. H., .. Fischl, B. (2002). Regional and progressive thinning of the cortical ribbon in Huntington's disease. Neurology, $58,695-701$.

Schacter, D. L., Harbluk, J. L., \& McLachlan, D. R. (1984). Retrieval without recollection: An experimental analysis of source amnesia. Journal of Verbal Learning and Verbal Behavior, 23, 593-611. doi:10.1016/s0022-5371(84)90373-6

Schacter, D. L., Kaszniak, A. W., Kihlstrom, J. F., \& Valdiserri, M. (1991). The relation between source memory and aging. Psychology and Aging, 6, 559-568. doi:10.1037/0882-7974.6.4.559 
Shallice, T. I. M., \& Burgess, P. W. (1991). Deficits in strategy application following frontal lobe damage in man. Brain, 114, 727-741. doi:10.1093/brain/114.2.727

Shapiro, A. M., Benedict, R. H., Schretlen, D., \& Brandt, J. (1999). Construct and concurrent validity of the Hopkins Verbal Learning Test-revised. The Clinical Neuropsychologist, 13, 348-358. doi:10.1076/clin.13.3.348.1749

Simons, J. S., Verfaellie, M., Galton, C. J., Miller, B. L., Hodges, J. R., \& Graham, K. S. (2002). Recollection-based memory in frontotemporal dementia: Implications for theories of long-term memory. Brain, 125, 2523-2536. doi:10.1093/brain/awf247

Slamecka, N. J., \& Graf, P. (1978). The generation effect: Delineation of a phenomenon. Journal of Experimental Psychology: Human Learning and Memory, 4, 592-604. doi:10.1037/0278-7393.4.6.592

Snowden, J. S., Craufurd, D., Thompson, J., \& Neary, D. (2002). Psychomotor, executive, and memory function in preclinical Huntington's disease. Journal of Clinical and Experimental Neuropsychology, 24, 133-145. doi:10.1076/jcen.24.2.133.998

Solomon, A. C., Stout, J. C., Johnson, S. A., Langbehn, D. R., Aylward, E. H., Brandt, J., . . Paulsen, J. S. (2007). Verbal episodic memory declines prior to diagnosis in Huntington's disease. Neuropsychologia , 45, 1767-1776. doi:10.1016/j.neuropsychologia.2006.12.015

Stout, J. C., Paulsen, J. S., Queller, S., Solomon, A. C., Whitlock, K. B., Campbell, J. C., . . Aylward, E. H. (2011). Neurocognitive signs in prodromal Huntington disease. Neuropsychology, 25, 1-14.

Tulving, E. (2002). Episodic memory: From mind to brain. Annual Review of Psychology, 53, 1-25. doi:10.1146/annurev.psych.53.100901.135114

Verny, C., Allain, P., Prudean, A., Malinge, M. C., Gohier, B., Scherer, C., ... Le Gall, D. (2007). Cognitive changes in asymptomatic carriers of the Huntington disease mutation gene. European Journal of Neurology, 14, 1344-1350. doi:10.1111/j.1468-1331.2007.01975.x

Wolf, R. C., Sambataro, F., Vasic, N., Wolf, N. D., Thomann, P. A., Landwehrmeyer, G. B., \& Orth, M. (2011). Longitudinal functional magnetic resonance imaging of cognition in preclinical Huntington's disease. Experimental neurology, 231, 214-222.

Received 3/ March 20 /4; revised version received 8 September 2014 